\title{
Existence of positive solutions for a discrete fractional boundary value problem
}

\author{
Jinhua Wang ${ }^{1 \dagger}$, Hongjun Xiang ${ }^{2^{*}+}$ and Fulai Chen ${ }^{1 \dagger}$
}

\section{"Correspondence:}

hunxhjxhj67@126.com

${ }^{2}$ The Editorial Department of

Journal of Xiangnan University, East

Wangxian Park, Chenzhou, 423000,

China

${ }^{\dagger}$ Equal contributors

Full list of author information is

available at the end of the article

\begin{abstract}
This paper is concerned with the existence of positive solutions to a discrete fractional boundary value problem. By using the Krasnosel'skii and Schaefer fixed point theorems, the existence results are established. Additionally, examples are provided to illustrate the effectiveness of the main results.
\end{abstract}

MSC: $26 \mathrm{~A} 33 ; 39 \mathrm{~A} 10 ; 47 \mathrm{H} 07$

Keywords: existence; positive solution; discrete; fractional boundary value problem

\section{Introduction}

Fractional differential equations have received increasing attention within the last ten years or so. The theory of fractional differential equations has been a new important mathematical branch due to its wide applications in different research areas and engineering, such as physics, chemistry, economics, control of dynamical etc. For more details, see [19] and the references therein. On the other hand, accompanied with the development of the theory for fractional calculus, fractional difference equations have attracted increasing attention slowly but steadily in the past three years or so. Some research papers have appeared, see [10-19]. For example, Atici and Eloe [10] analyzed the conjugate discrete fractional boundary value problem (FBVP) with delta derivative:

$$
\left\{\begin{array}{l}
-\Delta^{v} y(t)=f(t+v-1, y(t+v-1)), \quad t \in[0, b]_{\mathrm{N}_{0}} \\
y(v-2)=y(v+b+1)=0, \quad 1<v \leq 2
\end{array}\right.
$$

Goodrich [11] studied the discrete fractional boundary value problems:

$$
\left\{\begin{array}{l}
\Delta^{v} y(t)=\lambda f(t+v-1, y(t+v-1)), \quad t \in[0, T]_{Z}, \\
y(v-1)=y(v+T)+\sum_{i=1}^{N} F\left(t_{i}, y\left(t_{i}\right)\right), \quad 0<v<1 .
\end{array}\right.
$$

In [12], Lv discussed the existence of solutions for discrete fractional boundary value problems with a $p$-Laplacian operator:

$$
\left\{\begin{array}{l}
\Delta_{c}^{\beta}\left[\phi_{p}\left(\Delta_{c}^{\alpha} u\right)\right](t)=f(t+\alpha+\beta-1, u(t+\alpha+\beta-1)), \quad t \in[0, b]_{\mathrm{N}_{0}}, \\
\left.\Delta_{c}^{\alpha} u(t)\right|_{t=\beta-1}+\left.\Delta_{c}^{\alpha} u(t)\right|_{t=\beta+b}=0, \\
u(\alpha+\beta-2)+u(\alpha+\beta+b)=0, \quad 0<\alpha, \beta \leq 1,1<\alpha+\beta \leq 2
\end{array}\right.
$$

O2014 Wang et al.; licensee Springer. This is an Open Access article distributed under the terms of the Creative Commons Attribution License (http://creativecommons.org/licenses/by/2.0), which permits unrestricted use, distribution, and reproduction in any medium, provided the original work is properly cited. 
They obtained a series of excellent results of discrete fractional boundary value problems. Motivated by the aforementioned works, in this paper we consider a discrete fractional boundary value problem (FBVP):

$$
\left\{\begin{array}{l}
\Delta^{v} y(t)=f(t+v-1, y(t+v-1)), \quad t \in[0, b]_{\mathrm{N}_{0}} \\
y(v-2)=0, \quad \Delta y(v-2)=\Delta y(v+b-1)
\end{array}\right.
$$

where $1<v \leq 2, \Delta^{v}$ denotes the Riemann-Liouville fractional difference operator, $\mathbf{N}_{a}=$ $\{a, a+1, a+2, \ldots\}$ and $I_{\mathbf{N}_{a}}=I \cap \mathbf{N}_{a}$ for any number $a \in \mathbf{R}$ and each interval $I$ of $\mathbf{R}, b \in \mathbf{N}_{1}$. We appeal to the convention that $\sum_{s=k}^{k-1} y(s)=0$ for any $k \in \mathbf{N}_{a}$, where $y$ is a function defined on $\mathbf{N}_{a}$. By using the Krasnosel'skii and Schaefer fixed point theorems, the existence results are established and two examples are also provided to illustrate the effectiveness of the main results.

The rest of the paper is organized as follows. In Section 2, we introduce some lemmas and definitions which will be used later. In Section 3, the existence of positive solutions for the boundary value problem (1.1) is investigated. In Section 4, two examples are provided to illustrate the effectiveness of the main results.

\section{Basic definitions and preliminaries}

Firstly we present here some necessary definitions and lemmas which are used throughout this paper.

Definition 2.1 [13, 14] Define $t^{\underline{\nu}}:=\frac{\Gamma(t+1)}{\Gamma(t+1-v)}$ for any $t$ and $v$ for which the right-hand side is defined. If $t+1-v$ is a pole of the gamma function and $t+1$ is not a pole, then $t^{\underline{v}}=0$.

Definition 2.2 [15] The $v$ th fractional sum of a function $f$, for $v>0$, is defined to be

$$
\Delta^{-v} f(t)=\Delta^{-v} f(t ; a):=\frac{1}{\Gamma(v)} \sum_{s=a}^{t-v}(t-s-1)^{\frac{v-1}{f}} f(s)
$$

for $t \in\{a+v, a+v+1, \ldots\}:=\mathbf{N}_{a+v}$. Define the $v$ th fractional difference for $v>0$ by $\Delta^{v} f(t):=$ $\Delta^{N} \Delta^{v-N} f(t), t \in \mathbf{N}_{a+v}$ and $N \in \mathbf{N}$ satisfies $0 \leq N-1<v \leq N$.

Lemma 2.3 [15] Let $t$ and $v$ be any numbers for which $t^{\underline{v}}$ and $t^{\underline{v-1}}$ are defined. Then $\Delta t^{\underline{v}}=$ $v t^{\underline{\nu-1}}$.

Lemma 2.4 [15] Assume that $0 \leq N-1<v \leq N$. Then

$$
\Delta^{-v} \Delta^{v} y(t)=y(t)+C_{1} t^{\frac{v-1}{1}}+C_{2} t^{\frac{\nu-2}{2}}+\cdots+C_{N} t^{\frac{v-N}{}}
$$

for some $C_{i} \in \mathbf{R}$, with $1 \leq i \leq N$.

Lemma 2.5 (The nonlinear alternative of Leray and Schauder [20]) Let $\mathbf{E}$ be a Banach space with $C \subseteq \mathbf{E}$ closed and convex. Let $U$ be a relatively open subset of $C$ with $0 \in U$ and $T: \bar{U} \rightarrow C$ be a continuous and compact mapping. Then either

(a) the mapping $T$ has a fixed point in $\bar{U}$; or

(b) there exist $u \in \partial U$ and $\lambda \in(0,1)$ with $u=\lambda T u$. 
Lemma 2.6 [13] Let $\mathbf{B}$ be a Banach space and let $\mathbf{K} \subseteq \mathbf{B}$ be a cone. Assume that $\Omega_{1}$ and $\Omega_{2}$ are bounded open sets contained in $\mathbf{B}$ such that $0 \in \Omega_{1}$ and $\bar{\Omega}_{1} \subseteq \Omega_{2}$. Assume further that $T: \mathbf{K} \cap\left(\bar{\Omega}_{2} \backslash \Omega_{1}\right) \rightarrow \mathbf{K}$ is a completely continuous operator. If either

(i) $\|T y\| \leq\|y\|$ for $y \in \mathbf{K} \cap \partial \Omega_{1}$ and $\|T y\| \geq\|y\|$ for $y \in \mathbf{K} \cap \partial \Omega_{2}$; or

(ii) $\|T y\| \geq\|y\|$ for $y \in \mathbf{K} \cap \partial \Omega_{1}$ and $\|T y\| \leq\|y\|$ for $y \in \mathbf{K} \cap \partial \Omega_{2}$;

then $T$ has at least one fixed point in $\mathbf{K} \cap\left(\bar{\Omega}_{2} \backslash \Omega_{1}\right)$.

We state next the structural assumptions that we impose on (1.1).

$\left(\mathrm{H}_{1}\right)$ Assume that the nonlinearity function $f:[v-1, v+b-1]_{\mathbf{N}_{v-1}} \times \mathbf{R} \rightarrow[0,+\infty)$ is continuous.

$\left(\mathrm{H}_{2}\right)$ Assume that there exist nonnegative continuous functions $a_{1}(t), a_{2}(t), t \in[v-1, v+$ $b-1]_{\mathbf{N}_{v-1}}$ such that $|f(t, y)| \leq a_{1}(t)+a_{2}(t)|y|, \forall t \in[v-1, v+b-1]_{\mathbf{N}_{v-1}}, y \in \mathbf{R}$.

$\left(\mathrm{H}_{3}\right)$ Assume that $\lim _{y \rightarrow 0^{+}} \frac{f(t, y)}{y}=0$ uniformly for $t \in[v-1, v+b-1]_{\mathbf{N}_{v-1}}$.

$\left(\mathrm{H}_{4}\right)$ Assume that $\lim _{y \rightarrow+\infty} \frac{f(t, y)}{y}=+\infty$ uniformly for $t \in[v-1, v+b-1]_{\mathrm{N}_{v-1}}$.

\section{Existence results}

In this section, we will establish the existence of at least one positive solution for problem (1.1). At first, we state and prove some preliminary lemmas.

Lemma 3.1 Let $h:[v-1, v+b-1]_{\mathbf{N}_{v-1}} \rightarrow \mathbf{R}$ be given. Then the unique solution of the discrete fractional boundary value problem

$$
\left\{\begin{array}{l}
\Delta^{v} y(t)=h(t+v-1), \quad t \in[0, b]_{\mathrm{N}_{0}} \\
y(v-2)=0, \quad \Delta y(v-2)=\Delta y(v+b-1)
\end{array}\right.
$$

is

$$
y(t)=\frac{1}{\Gamma(v)} \sum_{s=0}^{b} G(t, s) h(s+v-1) .
$$

Here, for $(t, s) \in[v-2, v+b]_{\mathbf{N}_{v-2}} \times[0, b]_{\mathbf{N}_{0}}, G(t, s)$ is defined by

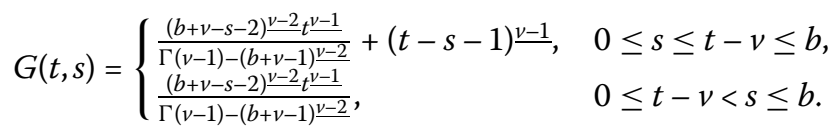

Proof Suppose that $y(t)$ defined on $[v-2, v+b]_{\mathbf{N}_{v-2}}$ is a solution of (3.1). Using Lemma 2.4, for some constants $C_{1}, C_{2} \in \mathbf{R}$, we have

$$
y(t)=\frac{1}{\Gamma(v)} \sum_{s=0}^{t-v}(t-s-1)^{\frac{\nu-1}{1}} h(s+v-1)+C_{1} t^{\frac{\nu-1}{}}+C_{2} t^{\frac{\nu-2}{2}}, \quad t \in[v-2, v+b]_{\mathbf{N}_{v-2}} .
$$

By $y(v-2)=0$ and Definition 2.1, we obtain $C_{2}=0$.

Then, for all $t \in[v-2, v+b-1]_{\mathrm{N}_{v-2}}$, we obtain [21]

$$
\Delta y(t)=\frac{1}{\Gamma(v-1)} \sum_{s=0}^{t-(v-1)}(t-s-1)^{\frac{v-2}{2}} h(s+v-1)+C_{1}(v-1) t^{\nu-2} .
$$


In view of $\Delta y(v-2)=\Delta y(v+b-1)$, we have

$$
C_{1}=\frac{1}{\Gamma(v-1)\left[\Gamma(v)-(v-1)(b+v-1)^{\left.\frac{v-2}{2}\right]}\right.} \sum_{s=0}^{b}(b+v-s-2)^{\frac{v-2}{2}} h(s+v-1) .
$$

Substituting the values of $C_{1}$ and $C_{2}$ in (3.4), we have

$$
\begin{aligned}
y(t)= & \frac{1}{\Gamma(v)} \sum_{s=0}^{t-v}(t-s-1)^{\frac{v-1}{2}} h(s+v-1) \\
& +\sum_{s=0}^{b} \frac{(b+v-s-2) \frac{v-2}{t} \frac{v-1}{\Gamma(v-1)\left[\Gamma(v)-(v-1)(b+v-1) \frac{v-2}{2}\right]} h(s+v-1)}{=} \frac{1}{\Gamma(v)} \sum_{s=0}^{b} G(t, s) h(s+v-1), \quad t \in[v-2, v+b-1]_{\mathbf{N}_{v-2}} .
\end{aligned}
$$

Lemma 3.2 The function $G(t, s)$ given in (3.3) satisfies the following:

(1) $0 \leq G(t, s) \leq \frac{D(v+b) \frac{v-1}{(s+v-1) \underline{v-1}}}{(2)}(s+v-1, s),(t, s) \in[v-2, v+b]_{\mathrm{N}_{v-2}} \times[0, b]_{\mathrm{N}_{0}}$;

(2) $\min _{t \in[v-1, v+b]_{\mathbf{N}_{v-1}}} G(t, s) \geq \frac{\Gamma(v)}{(s+v-1) \underline{v-1}} G(s+v-1, s)>0$.

Here,

$$
\begin{aligned}
D & =\max _{s \in[0, b]}\left\{1+\frac{\Gamma(v-1)-(v+b-1) \frac{v-2}{}}{(v+b-s-2) \frac{v-2}{}}\right\} \\
& =1+\frac{\Gamma(v-1)-(v+b-1)^{\frac{v-2}{2}}}{(v+b-2) \frac{v-2}{}} .
\end{aligned}
$$

Proof First of all, (3.3) implies that $G(s+v-1, s)=\frac{(v+b-s-2) \frac{v-2}{(s+v-1) \underline{v-1}}}{\Gamma(v-1)-(v+b-1)^{\frac{v-2}{}}}$. Note that $\Gamma(v-1)-$ $(v+b-1)^{\frac{v-2}{2}}>0$, we know $G(s+v-1, s)>0$.

Second of all, by (3.3) and the definition of $D$ in (3.6), we obtain

$$
\begin{aligned}
0 & \leq G(t, s) \leq \frac{(b+v-s-2)^{\frac{v-2}{2}}(v+b)^{\frac{v-1}{v}}}{\Gamma(v-1)-(b+v-1)^{\frac{v-2}{}}}+(v+b)^{\frac{v-1}{}} \\
& \leq \frac{D(b+v)^{\frac{v-1}{v-1}}}{(s+v-1)^{\frac{v-1}{2}}} G(s+v-1, s) .
\end{aligned}
$$

On the other hand,

$$
\begin{aligned}
& \min _{t \in[v-1, v+b]_{\mathbf{N}_{v-1}}} G(t, s)=\min _{t \in[v-1, v+b]_{\mathbf{N}_{v-1}}} \frac{(b+v-s-2)^{\frac{v-2}{t}} t^{\frac{v-1}{}}}{\Gamma(v-1)-(b+v-1)^{\frac{v-2}{2}}} \\
& \geq \frac{(v-1)^{\frac{v-1}{u}}}{(s+v-1)^{\frac{v-1}{}}} \cdot \frac{(b+v-s-2)^{\frac{v-2}{}(s+v-1)^{\frac{v-1}{}}}}{\Gamma(v-1)-(v+b-1)^{\frac{v-2}{}}} \\
& =\frac{\Gamma(v)}{(s+v-1) \underline{v-1}} G(s+v-1, s)>0 \text {. }
\end{aligned}
$$

The proof of Lemma 3.2 is completed.

Let $\mathbf{B}$ be the collection of all functions $y:[v-2, v+b]_{\mathbf{N}_{v-2}} \rightarrow \mathbf{R}$ with the norm $\|y\|=$ $\max \left\{|y(t)|: t \in[v-2, v+b]_{\mathbf{N}_{v-2}}\right\}$. 
Define the operator $T: \mathbf{B} \rightarrow \mathbf{B}$ by

$$
T y(t)=\frac{1}{\Gamma(v)} \sum_{s=0}^{b} G(t, s) f(s+v-1, y(s+v-1)) .
$$

In view of the continuity of $f$, it is easy to know that $T$ is continuous. Furthermore, it is not difficult to verify that $T$ maps bounded sets into bounded sets and equi-continuous sets. Therefore, in the light of the well-known Arzelá-Ascoli theorem, we know that $T$ is a compact operator (see $[11,12])$.

Let $\mathbf{E}=\left\{y \in \mathbf{B} \mid y(t) \geq 0, t \in[v-2, v+b]_{\mathbf{N}_{v-2}}\right\}$ and set

$$
\begin{aligned}
& A=\sum_{s=0}^{b} \frac{D(v+b)^{\frac{v-1}{-}} G(s+v-1, s)}{\Gamma(v)(s+v-1)^{\frac{v-1}{}}}\left\|a_{2}\right\|, \\
& B=\sum_{s=0}^{b} \frac{D(v+b)^{\frac{v-1}{L}} G(s+v-1, s)}{\Gamma(v)(s+v-1)^{\frac{v-1}{n}}}\left\|a_{1}\right\| .
\end{aligned}
$$

We have the following theorem.

Theorem 3.3 Assume that $\left(\mathrm{H}_{1}\right)$ and $\left(\mathrm{H}_{2}\right)$ hold. Then system (1.1) has at least one positive solution provided that

$$
\sum_{s=0}^{b} \frac{D(v+b)^{\underline{v-1}} G(s+v-1, s)}{\Gamma(v)(s+v-1) \underline{\underline{v-1}}}\left\|a_{2}\right\|<1 .
$$

Proof Let $\Omega=\{y \in \mathbf{E} \mid\|y\|<r\}$ with $r=\frac{B}{1-A}>0$. If $y \in \bar{\Omega}$, that is, $\|y\| \leq r$. From $\left(\mathrm{H}_{1}\right),\left(\mathrm{H}_{2}\right)$ and (3.9), we have

$$
\begin{aligned}
& \|T y(t)\|=\max _{t \in[v-2, v+b]_{\mathbf{N}-2}}\left|\frac{1}{\Gamma(v)} \sum_{s=0}^{b} G(t, s) f(s+v-1, y(s+v-1))\right| \\
& \leq \frac{1}{\Gamma(v)} \sum_{s=0}^{b} \frac{D(v+b)^{\frac{v-1}{\underline{v}}}}{(s+v-1) \underline{\underline{\nu}}} G(s+v-1, s)\left(\left|a_{1}(t)\right|+\left|a_{2}(t)\right||y(t)|\right) \\
& \leq \sum_{s=0}^{b} \frac{(v+b)^{\frac{v-1}{2}}}{\Gamma(v)} \frac{D G(s+v-1, s)}{(s+v-1)^{\frac{v-1}{\underline{ }}}}\left\|a_{1}\right\| \\
& +\sum_{s=0}^{b} \frac{(v+b)^{\frac{v-1}{}}}{\Gamma(v)} \frac{D G(s+v-1, s)}{(s+v-1) \underline{v-1}}\left\|a_{2}\right\|\|y\| \\
& =B+A\|y\| \leq r,
\end{aligned}
$$

which shows that $T y \in \bar{\Omega}$.

Consider the eigenvalue problem

$$
y=\lambda T y, \quad \lambda \in(0,1) .
$$


Assume that $y$ is a solution of (3.11), we obtain

$$
\|y\|=\|\lambda T y\|<\|T y\| \leq r
$$

It shows that $y \notin \partial \Omega$. By Lemma $2.5, T$ has a fixed point in $\bar{\Omega}$. The proof is completed.

We define the cone $\mathbf{K} \subseteq \mathbf{B}$ by

$$
\mathbf{K}=\left\{y \in \mathbf{E}: \min _{t \in[v-1, v+b]_{\mathbf{N}_{v-1}}} y(t) \geq \frac{\Gamma(v)}{D(v+b) \underline{v-1}}\|y\|\right\} .
$$

Lemma 3.4 Let $T$ be the operator defined in (3.9) and $\mathbf{K}$ be the cone defined in (3.13). Then $T: \mathbf{K} \rightarrow \mathbf{K}$.

Proof Note that for each $t \in[v-2, v+b]_{\mathbf{N}_{v-2}}$, we have

$$
\begin{aligned}
& \|T y(t)\|=\max _{t \in[v-2, v+b]_{\mathbf{N}_{v-2}}}\left|\frac{1}{\Gamma(v)} \sum_{s=0}^{b} G(t, s) f(s+v-1, y(s+v-1))\right| \\
& \leq \frac{1}{\Gamma(v)} \sum_{s=0}^{b} \frac{D(v+b) \underline{v-1}}{(s+v-1) \underline{v-1}} G(s+v-1, s)|f(s+v-1, y(s+v-1))| \\
& =\sum_{s=0}^{b} \frac{D(v+b)^{\frac{v-1}{-} G(s+v-1, s)}}{\Gamma(v)(s+v-1) \frac{v-1}{n}}|f(s+v-1, y(s+v-1))| .
\end{aligned}
$$

Therefore, it holds that

$$
\begin{aligned}
& \min _{t \in[v-1, v+b]_{\mathbf{N}_{v-1}}}(T y)(t) \\
& =\min _{t \in[v-1, v+b]_{\mathbf{N}_{v-1}}} \frac{1}{\Gamma(v)} \sum_{s=0}^{b} G(t, s) f(s+v-1, y(s+v-1)) \\
& \geq \frac{1}{\Gamma(v)} \sum_{s=0}^{b} \frac{\Gamma(v)}{(s+v-1) \frac{v-1}{n}} G(s+v-1, s) f(s+v-1, y(s+v-1)) \\
& =\frac{\Gamma(v)}{D(v+b) \underline{v-1}} \sum_{s=0}^{b} \frac{D(v+b) \frac{v-1}{\Gamma(v)(s+v-1) \frac{v-1}{n}} f(s+v-1, y(s+v-1))}{\geq} \\
& \quad \frac{\Gamma(v)}{D(v+b) \underline{v-1}}\|T y\| .
\end{aligned}
$$

The conclusion of Lemma 3.4 holds.

Theorem 3.5 Suppose that conditions $\left(\mathrm{H}_{1}\right),\left(\mathrm{H}_{3}\right)$ and $\left(\mathrm{H}_{4}\right)$ hold. Then problem (1.1) has at least one positive solution.

Proof We have already shown $T(\mathbf{K}) \subseteq \mathbf{K}$ in Lemma 3.4. By condition $\left(\mathrm{H}_{3}\right)$, we can select $\eta_{1}>0$ sufficiently small so that both $|f(t, y)| \leq \eta_{1}\|y\|$ and $\eta_{1} \sum_{s=0}^{b} \frac{(v+b) \frac{v-1}{\Gamma(v)}}{(s+v-1)^{\underline{v-1}}}<1$ hold for all $t \in[v-1, v+b-1]_{\mathbf{N}_{v-1}}$ and $0<y<r_{1}$, where $r_{1}:=r_{1}\left(\eta_{1}\right)$. 
Let $\Omega_{1}=\left\{y \in \mathbf{B}:\|y\|<r_{1}\right\}$. Then, for $y \in \partial \Omega_{1} \cap \mathbf{K}$, we have

$$
\begin{aligned}
& \|T y(t)\|=\max _{t \in[v-2, v+b]_{\mathbf{N}_{v-2}}}\left|\frac{1}{\Gamma(v)} \sum_{s=0}^{b} G(t, s) f(s+v-1, y(s+v-1))\right| \\
& \leq \frac{1}{\Gamma(v)} \sum_{s=0}^{b} \frac{D(v+b)^{\frac{v-1}{v}}}{(s+v-1)^{\frac{v-1}{n}}} G(s+v-1, s)|f(s+v-1, y(s+v-1))| \\
& \leq \sum_{s=0}^{b} \frac{D(v+b)^{\frac{v-1}{}} G(s+v-1, s)}{\Gamma(v)(s+v-1)^{\frac{v-1}{2}}} \eta_{1}\|y\|<\|y\| .
\end{aligned}
$$

It implies that $T$ is a cone contraction on $y \in \partial \Omega_{1} \cap \mathbf{K}$.

On the other hand, from condition $\left(\mathrm{H}_{4}\right)$, we may select a number $\eta_{2}>0$ such that both $|f(t, y)|>\eta_{2}\|y\|$ and $\eta_{2} \sum_{s=0}^{b} \frac{G(s+v-1, s)}{(s+v-1) \frac{v-1}{-}}>1$ hold for all $t \in[v-1, v+b-1]_{\mathbf{N}_{v-1}}$ and $0<y<r_{2}$, where $r_{2}:=r_{2}\left(\eta_{2}\right)$ and $r_{2}>r_{1}>0$. Define $\Omega_{2}=\left\{y \in \mathbf{B}:\|y\|<r_{2}\right\}$, we obtain

$$
\begin{aligned}
\|T y(t)\| & =\max _{t \in[v-2, v+b]_{\mathbf{N}_{v-2}}} \frac{1}{\Gamma(v)} \sum_{s=0}^{b} G(t, s)|f(s+v-1, y(s+v-1))| \\
& \geq \frac{1}{\Gamma(v)} \sum_{s=0}^{b} \min _{t \in[v-1, v+b]_{\mathbf{N}_{v-1}}} G(t, s)|f(s+v-1, y(s+v-1))| \\
& >\sum_{s=0}^{b} \frac{G(s+v-1, s)}{(s+v-1) \frac{v-1}{v}} \eta_{2}\|y\|>\|y\|,
\end{aligned}
$$

whenever $y \in \partial \Omega_{2} \cap \mathbf{K}$, so that $T$ is a cone expansion on $\partial \Omega_{2} \cap \mathbf{K}$.

In summary, we may invoke Lemma 2.6 to deduce the existence of a function $y_{0} \in \mathbf{K} \cap$ $\left(\bar{\Omega}_{2} \backslash \Omega_{1}\right)$ such that $T y_{0}=y_{0}$, where $y_{0}$ is a positive solution to problem (1.1). The proof is completed.

\section{Example}

Example 4.1 Consider the fractional difference boundary value problem

$$
\left\{\begin{array}{l}
\Delta^{\frac{3}{2}} y(t)=f\left(t+\frac{1}{2}, y\left(t+\frac{1}{2}\right)\right), \quad t \in[0,3]_{\mathrm{N}_{0}}, \\
y\left(-\frac{1}{2}\right)=0, \quad \Delta y\left(-\frac{1}{2}\right)=\Delta y\left(\frac{7}{2}\right)
\end{array}\right.
$$

Set $a_{1}(t)=1, a_{2}(t)=\frac{t}{30}, f(t, y)=\frac{t|y|}{30}+|\sin t|, t \in\left[\frac{1}{2}, \frac{7}{2}\right]_{\mathbf{N}_{\frac{1}{2}}}$. We have

$$
|f(t, y)| \leq 1+\frac{t}{30}|y|
$$

By a simple computation, we can obtain $D \approx 1.7750, \sum_{s=0}^{3} \frac{\left(\frac{9}{2}\right)^{\frac{1}{2}} G\left(s+\frac{1}{2}, s\right)}{\Gamma\left(\frac{3}{2}\right)\left(s+\frac{1}{2}\right)^{\frac{1}{2}}} \approx 4.7688,\left\|a_{2}\right\|=$ $\frac{7}{60}$. Therefore, $A \approx 0.9875<1$. The conditions of Theorem 3.3 hold, the boundary value problem (4.1) has at least one positive solution. 
Example 4.2 Consider the fractional difference boundary value problem

$$
\left\{\begin{array}{l}
\Delta^{\frac{3}{2}} y(t)=f\left(t+\frac{1}{2}, y\left(t+\frac{1}{2}\right)\right), \quad t \in[0,11]_{\mathrm{N}_{0}} \\
y\left(-\frac{1}{2}\right)=0, \quad \Delta y\left(-\frac{1}{2}\right)=\Delta y\left(\frac{23}{2}\right) .
\end{array}\right.
$$

Set $f(t, y)=t y^{2}, t \in\left[\frac{1}{2}, \frac{23}{2}\right]_{\mathbf{N}_{\frac{1}{2}}}$. We have

$$
\text { (1) } \lim _{y \rightarrow 0^{+}} \frac{f(t, y)}{y}=\lim _{y \rightarrow 0^{+}} \frac{t y^{2}}{y}=0, \quad \text { (2) } \quad \lim _{y \rightarrow+\infty} \frac{f(t, y)}{y}=\lim _{y \rightarrow+\infty} \frac{t y^{2}}{y}=+\infty \text {. }
$$

The conditions of Theorem 3.5 hold, the boundary value problem (4.2) has at least one positive solution.

\section{Competing interests}

The authors declare that they have no competing interests.

\section{Authors' contributions}

The authors declare that the study was realized in collaboration with the same responsibility. All authors read and approved the final manuscript.

\section{Author details}

${ }^{1}$ Department of Mathematics, Xiangnan University, East Wangxian Park, Chenzhou, 423000, China. ${ }^{2}$ The Editorial Department of Journal of Xiangnan University, East Wangxian Park, Chenzhou, 423000, China.

\section{Acknowledgements}

This work was jointly supported by the Natural Science Foundation of China under Grants 11471278, the Natural Science Foundation of Hunan Province under Grants 13JJ3120 and 14JJ2133, and the Construct Program of the Key Discipline in Hunan Province. We would like to show our great thanks to the anonymous referee for his/her valuable suggestions and comments, which improve the former version of this paper and make us rewrite the paper in a more clear way.

Received: 18 April 2014 Accepted: 9 September 2014 Published: 25 Sep 2014

\section{References}

1. Samko, SG, Kilbas, AA, Marichev, Ol: Fractional Integrals and Derivatives. Theory and Applications. Gordon \& Breach, Yverdon (1993)

2. Podlubny, l: Fractional Differential Equations. Mathematics in Science and Engineering, vol. 198. Academic Press, New York (1999)

3. Bai, ZB, Lü, HS: Positive solutions for boundary value problem of nonlinear fractional differential equation. J. Math Anal. Appl. 311, 495-505 (2005)

4. Zhang, SQ: Positive solutions for boundary value problems of nonlinear fractional differential equations. Electron. J. Differ. Equ. 2006, 36 (2006)

5. Jafari, H, Gejji, VD: Positive solutions of nonlinear fractional boundary value problems using Adomian decomposition method. Appl. Math. Comput. 180, 700-706 (2006)

6. Dehghani, R, Ghanbari, K: Triple positive solutions for boundary value problem of a nonlinear fractional differential equation. Bull. Iran. Math. Soc. 33(2), 1-14 (2007)

7. Wang, JH, Xiang, HJ, Liu, ZG: Positive solutions for three-point boundary value problems of nonlinear fractional differential equations with P-Laplacian. Far East J. Appl. Math. 37(1), 33-47 (2009)

8. Wang, JH, Xiang, HJ, Liu, ZG: Upper and lower solutions method for a class of singular fractional boundary value problems with P-Laplacian. Abstr. Appl. Anal. 2010, Article ID 971824 (2010)

9. Cai, G: Positive solutions for boundary value problems of fractional differential equations with $P$-Laplacian operator. Bound. Value Probl. 2012, Article ID 18 (2012)

10. Atici, FM, Eloe, PW: Two-point boundary value problems for finite fractional difference equations. J. Differ. Equ. Appl. $17,445-456(2011)$

11. Goodrich, CS: On a first-order semipositone discrete fractional boundary value problem. Arch. Math. 99, 509-518 (2012)

12. LV, WD: Existence of solutions for discrete fractional boundary value problems with a P-Laplacian operator. Adv. Differ. Equ. 2012, Article ID 163 (2012)

13. Goodrich, CS: Existence of a positive solution to a system of discrete fractional boundary value problems. Appl. Math Comput. 217, 4740-4753 (2011)

14. Atici, FM, Eloe, PW: A transform method in discrete fractional calculus. Int. J. Differ. Equ. 2(2), 165-176 (2007)

15. Goodrich, CS: On discrete sequential fractional boundary value problems. J. Math. Anal. Appl. 385, 111-124 (2012)

16. Goodrich, CS: Positive solutions to boundary value problems with nonlinear boundary conditions. Nonlinear Anal. 75 417-432 (2012) 
17. Goodrich, CS: On discrete fractional three-point boundary value problems. J. Differ. Equ. Appl. 18, 397-415 (2012)

18. Goodrich, CS: Nonlocal systems of BVPs with asymptotically superlinear boundary conditions. Comment. Math. Univ. Carol. 53, 79-97 (2012)

19. Atici, FM, Sengul, S: Modeling with fractional difference equations. J. Math. Anal. Appl. 369, 1-9 (2010)

20. Zeidler, E: Nonlinear Functional Analysis and Applications, l: Fixed Point Theorems. Springer, New York (1986)

21. Atici, FM, Eloe, PW: Initial value problems in discrete fractional calculus. Proc. Am. Math. Soc. 137, $981-989$ (2009)

10.1186/1687-1847-2014-253

Cite this article as: Wang et al.: Existence of positive solutions for a discrete fractional boundary value problem. Advances in Difference Equations 2014, 2014:253

Submit your manuscript to a SpringerOpen ${ }^{\circ}$ journal and benefit from:

- Convenient online submission

- Rigorous peer review

- Immediate publication on acceptance

- Open access: articles freely available online

- High visibility within the field

- Retaining the copyright to your article 